\title{
Crank-Nicholson Scheme of the Zeroth-Order Approximate Deconvolution Model of Turbulence Based On a Mixed Formulation
}

\author{
Mustafa Aggul \\ 0000-0003-4013-9907 \\ Department of Mathematics, Hacettepe University, 06800, Ankara, Turkey
}

\begin{abstract}
This report presents a method with high spatial and temporal accuracy for estimating solutions of Navier-Stokes equations at high Reynolds number. It employs Crank-Nicolson time discretization along with the zeroth-order approximate deconvolution model of turbulence to regularize the flow problem; solves a deviation of the Navier Stokes equation instead. Both theoretical and computational findings of this report illustrate that the model produces a high order of accuracy and stability. Furthermore, measurements of the drag and lift coefficients of a benchmark problem verify the potential of the model in this kind of computations.
\end{abstract}

Keywords: Approximate deconvolution; Crank-Nicholson; Stability; Accuracy; Turbulence models; Space filter

\author{
* Corresponding author \\ Mustafa Aggul \\ mustafaaggul@hacettepe.edu.tr
}

Adress: Department of Mathematics, Hacettepe University, 06800, Anka$\mathrm{ra}$, Turkey

Tel: +903122977870

Fax: +903122992017

Research Article

Manuscript

Received 29.04.2020

Revised 23.06.2020

Accepted 02.07.2020

Doi: 10.30939/ijastech..729443

\section{Introduction}

Navier-Stokes equations represent the motion of the fluid flow. In the incompressible flow case, it seeks for the velocity-pressure pair $u: \Omega \times[0, T] \rightarrow R^{d}(d=2,3)$ and $p: \Omega \times(0, T] \rightarrow R$ satisfying

$$
\begin{gathered}
u_{t}+u \cdot \nabla u-v \Delta u+\nabla p=f, \text { for } x \in \Omega, 0<t \leq T, \\
\nabla \cdot u=0, x \in \Omega, \text { for } 0 \leq t \leq T, \\
u(x, 0)=u_{0}(x), \text { for } x \in \Omega,
\end{gathered}
$$

for $0<t \leq T$. Throughout this paper, for theoretical reasoning, we consider the case of periodic boundary conditions. Nevertheless, numerical experiments with Dirichlet boundary conditions still performs as expected.

Its well-known that fluid flow is continuum of scales, where the smallest persistent scales in the case of a 3D flow is of the order $\mathcal{O}\left(R e^{-3 / 4}\right)$ in size where the Reynolds number $R e$ is inverse proportional to the viscosity coefficient $v$. Considering real-life applications of this equation, Re could easily be around $\sim \mathcal{O}\left(10^{10}\right)$ and even more for large domains, e.g., for atmosphere-ocean interaction. Capturing all persistent scales, in many applications is not feasible or indeed impossible. Contrarily, the large scales in the flow are responsible for much of the mixing and most of the momentum transport. Upon this observation, turbulence models like Large Eddy Simulation (LES) gained much attention $[1,2,3]$. The idea is that set a filter width $\delta>0$ for the size of large scales and model the effect of the smaller scales to these large scales. This merely requires solving for large scales (bigger than $\delta$ ), and hence avoids singularities due to small turbulent fluctuations.

If $(\cdot)^{\delta}$ denotes a local, space averaging operator which commutes with differentiation, then averaging (1) gives the following non-closed equations for $\bar{u}^{\delta}$ and $\bar{p}^{\delta}$ in $(0, T) \times$ $\Omega$ :

$$
\begin{gathered}
\bar{u}_{t}^{\delta}+\nabla \cdot\left(\bar{u}^{\delta}{\overline{u^{T}}}^{\delta}\right)-v \Delta \bar{u}^{\delta}+\nabla \bar{p}^{\delta} \\
+\nabla \cdot\left({\overline{u u^{T}}}^{\delta}-\bar{u}^{\delta} \bar{u}^{T}\right)=\bar{f}^{\delta} \\
\nabla \cdot \bar{u}^{\delta}=0 .
\end{gathered}
$$

An LES model is required when one tries to close the system (2) by choosing an approximation to the last term on the left-hand side of (2a). In this report, we consider the family of Approximate Deconvolution Models (ADMs). These models were introduced by Stolz and Adams in [4] and further studied in e.g., [5-10]. Given the family of approximate deconvolution operators $G_{N}$ (explained in Section 2), the ADM for Navier-Stokes equations is, with the new model variables $(\mathrm{w}, \mathrm{q})$, as follows:

$$
w_{t}+\nabla \cdot{\overline{\left(G_{N} w\right)\left(G_{N} w\right)^{T}}}^{\delta}-v \Delta w+\nabla \bar{q}^{\delta}=\bar{f}^{\delta},
$$




$$
\nabla \cdot w=0
$$

with $w(0, \mathrm{x})=\overline{\mathrm{u}}_{0}^{\delta}(\mathrm{x})$ and periodic boundary conditions (with zero means).

We employ the zeroth-order approximate deconvolution model of turbulence; see, e.g., [6]. Throughout this paper, we use the self-adjoint filtering operator $\mathrm{A}^{-1}=(\mathrm{I}-$ $\left.\delta^{2} \Delta\right)^{-1}$ detailed in Section 2.

The zeroth-order $(N=0)$ ADM emerges when exact deconvolution $A$ is applied to both sides of $(1.3 a)$. In the variational formulation: seek $(w, q) \in\left(\left(X \cap H^{2}(\Omega)\right), Q\right)$ such that for any $(v, \chi) \in(X, Q)$

$$
\begin{gathered}
\left(w_{t}, v\right)+\delta^{2}\left(\nabla w_{t}, \nabla v\right)+v(\nabla w, \nabla v)+v \delta^{2}(\Delta w, \Delta v) \\
+b^{*}(w, w ; v)-(q, \nabla \cdot v)=(f, v) \\
(\nabla \cdot w, \chi)=0
\end{gathered}
$$

However, this choice of the filtering operator results in a fourth order term $v \delta^{2}(\Delta w, \Delta v)$ in (4), which require employing $\mathrm{C}^{1}$ elements as is. Therefore, we follow [6] and use the mixed variational formulation: seek $(w, \zeta, q) \in$ $(X, X, Q)$ such that for any $(v, \xi, \chi) \in(X, X, Q)$

$$
\begin{gathered}
\left(w_{t}, v\right)+\delta^{2}\left(\nabla w_{t}, \nabla v\right)+v(\nabla w, \nabla v)+v \delta^{2}(\nabla \zeta, \nabla v) \\
+b^{*}(w, w ; v)-(q, \nabla \cdot v)=(f, v) \\
(\nabla w, \nabla \xi)=(\zeta, \xi) \\
(\nabla \cdot w, \chi)=0
\end{gathered}
$$

The spaces $X$ and $Q$ are defined in Section 2.

Zeroth-order ADM attains high spatial accuracy. However, time accuracy is also crucial for turbulence modeling. Accordingly, it shall be supplied with a stable high accuracy time discretization. This goal has been addressed with a predictor-corrector type deferred correction method in [11] and [12]. In this report, we investigate Crank-Nicolson scheme employed for zeroth-order ADM (CN-ADM).

The report is organized as follows. In Section 2, we introduce the necessary notations and preliminary results. The new method is introduced, and the stability and accuracy results are given in section 3 . The numerical tests involving a quantitative and a qualitative assessment will be presented in Section 4.

\section{Mathematical Preliminaries and Notations}

Throughout this paper, the norm $\|\cdot\|$ denotes the usual $\mathrm{L}^{2}(\Omega)$-norm of scalars, vectors, and tensors, induced by the usual $L^{2}$ inner-product, denoted by $(\cdot, \cdot)$.

The space that the velocity (at time $t$ ) will be sought in is given by

$$
\begin{gathered}
X=H_{\text {per }}^{1}(\Omega)^{d}=\left\{v \in L^{2}(\Omega)^{d}: \nabla v \in L^{2}(\Omega)^{d \times d}\right. \\
\text { and } v \text { periodic with period } L\}
\end{gathered}
$$

equipped with the norm $\|v\|_{X}=\|\nabla v\|$. The space dual to $X$ has the norm

$$
|f|_{-1}=\sup _{v \in X} \frac{(f, v)}{\|\nabla v\|}
$$

The pressure (at time $t$ ) is sought in the space

$$
Q=L_{p e r}^{2}(\Omega)=\left\{q: q \in L^{2}(\Omega), \int_{\Omega} q(x) d x=0,\right.
$$

and $q$ periodic with period $L\}$.

Also, the space of weakly divergence-free functions is $\mathrm{V}$, a subset of $\mathrm{X}$, defined as

$$
V=\{v \in X:(\nabla \cdot v, q)=0, \forall q \in Q\}
$$

For measurable function $v:[0, T] \rightarrow X$, we define

$$
\|v\|_{L} p_{(0, T ; X)}=\left(\int_{0}^{T}\|v(t)\|_{X}^{p} d t\right)^{\frac{1}{p}} d t, 1 \leq p<\infty
$$

and

$$
\|v\|_{L^{\infty}(0, T ; X)}=e s s \sup _{0 \leq t \leq T}\|v(t)\|_{X} .
$$

We assume throughout the paper that the velocitypressure finite element spaces $X^{h} \subset X$ and $Q^{h} \subset Q$ are conforming, have common approximation properties of finite element spaces, and also satisfy the discrete inf-sup, or $L B B^{h}$, condition

$$
\inf _{q^{h} \in Q^{h}} \sup _{v^{h} \in X^{h}} \frac{\left(q^{h}, \nabla \cdot v^{h}\right)}{\left\|\nabla v^{h}\right\| \cdot\left\|q^{h}\right\|} \geq \beta^{h}>0,
$$

where $\beta^{h}$ is bounded away from zero uniformly in $h$. Examples of such spaces can be found in [13]. A commonly used examples of these spaces are $X^{h} \subset X$, $Q^{h} \subset Q$, involving continuous piecewise polynomials of degree $r$ and $r-1$, respectively, with $r \geq 1$.

The space of discretely divergence-free functions is

$$
V^{h}=\left\{v^{h} \in X^{h}:\left(q^{h}, \nabla \cdot v^{h}\right)=0, \forall q^{h} \in Q^{h}\right\} .
$$

The approximate deconvolution model of turbulence is constructed on the following operator.

\section{Definition 1 (Approximate Deconvolution Operator)}

For a fixed finite $N$, define the $N t h$ approximate deconvolution operator $G_{N}$ by

$$
G_{N} \phi=\sum_{n=0}^{N}\left(I-A_{\delta}^{-1}\right)^{n} \phi,
$$

where the averaging operator $A_{\delta}^{-1}$ is the differential filter: given $\phi \in L^{2}(\Omega), \bar{\phi}^{\delta} \in H^{2}(\Omega)$ is the unique solution of

$$
A_{\delta \bar{\phi}}^{\delta}:=-\delta^{2} \Delta \bar{\phi}^{\delta}+\bar{\phi}^{\delta}=\phi \quad \text { in } \Omega
$$


under periodic boundary conditions. With periodicity assumption on boundaries, this operator commutes with differentiation.

Lemma 2 The operator $G_{N}^{i}$ is compact, positive, and is an asymptotic inverse to the filter $A_{\delta}^{-1}$, i.e., for very smooth $\phi$ and as $\delta \rightarrow 0$, it satisfies

$$
\phi=G_{N} \bar{\phi}^{\delta}+(-1)^{N+1} \delta^{2 N+2} \Delta^{N+1} A_{\delta}^{-(N+1)} \phi .
$$

The proof of the lemma can be found in [10].

We also define the following norm, induced by the deconvolution operator $A$ :

$$
|\phi|_{A}^{2}=|\phi|^{2}+\delta^{2}|\nabla \phi|^{2} .
$$

In the analysis we use the properties of the following Modified Stokes Projection (see [6]).

\section{Definition 3 (Modified Stokes Projection)}

Define the Stokes projection operator

$$
\begin{gathered}
P_{S}:(X, X, Q) \rightarrow\left(X^{h}, X^{h}, Q^{h}\right), \\
P_{S}(u, \zeta, p)=(\tilde{u}, \tilde{\zeta}, \tilde{p}),
\end{gathered}
$$

satisfying

$$
\begin{gathered}
v\left(\nabla(u-\tilde{u}), \nabla v^{h}\right)+v \delta^{2}\left(\nabla(\zeta-\tilde{\zeta}), \nabla v^{h}\right) \\
+\left(p-\tilde{p}, \nabla \cdot v^{h}\right)=0, \\
\left(\nabla(u-\tilde{u}), \nabla \xi^{h}\right)=\left(\zeta-\tilde{\zeta}, \xi^{h}\right), \\
\left(\nabla \cdot(u-\tilde{u}), q^{h}\right)=0,
\end{gathered}
$$

for any $v^{h} \in X^{h}, \xi^{h} \in X^{h}, q^{h} \in Q^{h}$.

In $\left(V^{h}, X^{h}, Q^{h}\right)$, this formulation reads: given

$$
\begin{gathered}
(u, \zeta, p) \in(V, X, Q), \text { find }(\tilde{u}, \tilde{\zeta}) \in\left(V^{h}, X^{h}\right) \text { satisfying } \\
\begin{array}{c}
v\left(\nabla(u-\tilde{u}), \nabla v^{h}\right)+v \delta^{2}\left(\nabla(\zeta-\tilde{\zeta}), \nabla v^{h}\right) \\
+\left(p-q^{h}, \nabla \cdot v^{h}\right)=0 \\
\left(\nabla(u-\tilde{u}), \nabla \xi^{h}\right)=\left(\zeta-\tilde{\zeta}, \xi^{h}\right)
\end{array}
\end{gathered}
$$

for any $v^{h} \in V^{h}, \xi^{h} \in X^{h}, q^{h} \in Q^{h}$.

We give the stability and accuracy results for the modified Stokes projection (9) without proof. The proof can be found in [6]. The proof requires the inverse inequality to hold

$$
\|\nabla \phi\| \leq C h^{-1}\|\phi\|, \forall \phi \in X^{h} .
$$

To that end, we assume the mesh to be quasi-uniform.

\section{Preposition 4 (Stability of the Stokes Projection)}

Let $\tilde{u}, \tilde{\zeta}$ satisfy (9) for given $u, \zeta$. The following bound holds:

$$
\begin{aligned}
& v\|\nabla \tilde{u}\|^{2}+v \delta^{2}\|\tilde{\zeta}\|^{2} \\
& \leq C\left(v\left(1+\delta^{2} h^{-2}\right)\|\nabla u\|^{2}+v \delta^{2}\|\zeta\|^{2}\right. \\
&+v^{-1} \inf _{q^{h} \in Q^{h}}\left\|p-q^{h}\right\|^{2}
\end{aligned}
$$

In the error analysis of the proposed method, we use the error estimate of the Stokes projection (9).

\section{Preposition 5 (Error estimate for the Stokes Projection)}

Suppose the discrete inf-sup condition (8) holds. Then, the error in the Stokes projection satisfies

$$
\begin{aligned}
& v\|\nabla(u-\tilde{u})\|^{2}+v \delta^{2}\|\zeta-\widetilde{\zeta}\|^{2} \\
& \leq C\left[v\left(1+\delta^{2} h^{-2}\right) \inf _{v^{h} \in V^{h}}\left\|\nabla\left(u-v^{h}\right)\right\|^{2}\right. \\
& +v \delta^{2}\left(1+\delta^{2} h^{-2}\right) \inf _{\xi^{h} \in X^{h}}\left\|\zeta-\xi^{h}\right\|^{2} \\
& +v^{-1} \inf _{q^{h} \in Q^{h}}\left\|p-q^{h}\right\|^{2}
\end{aligned}
$$

where $C$ is a constant independent of $h$ and $R e$.

Define the explicitly skew-symmetrized trilinear form

$$
b^{*}(u, v, w):=\frac{1}{2}(u \cdot \nabla v, w)-\frac{1}{2}(u \cdot \nabla w, v) .
$$

The following estimates are commonly used (see [13] for proofs):

There exists a constant $C=C(\Omega)$ such that

$\left|b^{*}(u, v, w)\right| \leq C(\Omega) \quad\|\nabla u\|\|\nabla v\|\|\nabla w\|$,

$\left|b^{*}(u, v, w)\right| \leq C(\Omega) \sqrt{\|u\|\|\nabla u\|}\|\nabla v\|\|\nabla w\|$.

We will also need the following inequalities: for any $\mathrm{u} \in \mathrm{V}$

$$
\begin{array}{r}
\inf _{v \in V^{h}}|\nabla(u-v)| \leq C(\Omega) \inf _{v \in X^{h}}|\nabla(u-v)| \\
\inf _{v \in V^{h}}|u-v| \leq C(\Omega) \inf _{v \in X^{h}}|\nabla(u-v)|,
\end{array}
$$

The proof of (10) can be found in [13], and (11) follows from the Poincare-Friedrich's inequality and (10).

Through this report, the averages of two time levels are denoted by $v^{n+1 / 2}=\frac{1}{2}\left(v^{n+1}+v^{n}\right)$. Also let $v^{n}=$ $v\left(t_{n}\right)$ and let $v^{h, n}$ approximate $v^{n}$ at $t_{n}$ time level.

\section{Formulation and Theoretical Results}

\section{Algorithm 6}

Let $f \in L^{2}\left(0, T ; H^{-1}(\Omega)\right)$, time step $\Delta t>0$ and end time $T>0$ be given. Set $M=T / \Delta t$ and $w^{h, 0}=$ $\bar{u}(0), q^{h, 0}=p(0)$. For all 
$n=0,1, \ldots, M-1$, compute $w^{h, n+1}, q^{h, n+1}$ via:

Find $w^{h, n+1}, \zeta^{h, n+1} \in X_{h}, q^{h, n+1} \in Q_{h}$ satisfying for all $v^{h}, \xi^{h} \in X_{h}, \chi^{h} \in Q_{h}$

$$
\begin{gathered}
\left(\frac{w^{h, n+1}-w^{h, n}}{\Delta t}, v^{h}\right)+\delta^{2}\left(\nabla\left(\frac{w^{h, n+1}-w^{h, n}}{\Delta t}\right), \nabla v^{h}\right) \\
+v\left(\nabla w^{h, n+1 / 2}, \nabla v^{h}\right)+v \delta^{2}\left(\nabla \zeta^{h, n+1 / 2}, \nabla v^{h}\right) \\
+b^{*}\left(w^{h, n+1 / 2}, w^{h, n+1 / 2}, v^{h}\right)-\left(q^{h, n+1 / 2}, \nabla \cdot v^{h}\right) \\
=\left(\frac{f\left(t_{n+1}\right)+f\left(t_{n}\right)}{2}, v^{h}\right), \\
\left(\nabla w^{h, n+1 / 2}, \nabla \xi^{h}\right)=\left(\zeta^{h, n+1 / 2}, \xi^{h}\right), \\
\left(\nabla \cdot w^{h, n+1 / 2}, \chi^{h}\right)=0 .
\end{gathered}
$$

\section{Theorem 7 (Stability)}

Let $w^{h, n+1}$ be computed by Algorithm 6. Let $f \in$ $L^{2}\left(0, T ; H^{-1}(\Omega)\right)$. Then, for $n=0, \ldots, M-1$,

$$
\begin{gathered}
\left|w^{h, M}\right|_{A}^{2}+v \Delta t \sum_{n=0}^{M-1} \|\left.\nabla w^{h, n+1 / 2}\right|^{2} \\
\quad 2 v \delta^{2} \Delta t \sum_{n=0}^{M-1}|| \zeta^{h, n+1 / 2} \|^{2} \leq\left|w^{h, 0}\right|_{A}^{2} \\
\quad+v^{-1} \Delta t \sum_{n=0}^{M-1}\left|\frac{f\left(t_{n+1}\right)+f\left(t_{n}\right)}{2}\right|_{-1}^{2} .
\end{gathered}
$$

Proof. Take $v^{h}=w^{h, n+1 / 2} \in X_{h}$ in (12), $\xi^{h}=$ $\zeta^{h, n+1 / 2} \in X_{h}$ in (13) and $\chi^{h}=q^{h, n+1 / 2}$ in (14), and then substitute (13) and (14) in (12). Due to the skewsymmetry property of the trilinear form

$b^{*}\left(w^{h, n+1 / 2}, w^{h, n+1 / 2}, w^{h, n+1 / 2}\right)=0$ and

$\left(q^{h, n+1 / 2}, \nabla \cdot w^{h, n+1 / 2}\right)=0$ from (14). Therefore,

$$
\begin{aligned}
& \frac{1}{2 \Delta t}\left(\left|w^{h, n+1}\right|_{A}-\left|w^{h, n}\right|_{A}\right) \\
& +v\left\|\nabla w^{h, n+1 / 2}\right\|^{2}+v \delta^{2}|| \zeta^{h, n+1 / 2} \|^{2} \\
& \leq\left(\frac{f\left(t_{n+1}\right)+f\left(t_{n}\right)}{2}, w^{h, n+1 / 2}\right) .
\end{aligned}
$$

Using the definition of $H^{-1}(\Omega)$-norm on the right-hand side and applying Young's inequality

$$
\begin{aligned}
&\left(\frac{f\left(t_{n+1}\right)+f\left(t_{n}\right)}{2}\right.\left., w^{h, n+1 / 2}\right) \\
& \leq\left|\frac{f\left(t_{i+1}\right)+f\left(t_{i}\right)}{2}\right|_{-1}|| \nabla w^{h, n+1 / 2} \| \\
& \leq \frac{1}{2 v}\left|\frac{f\left(t_{i+1}\right)+f\left(t_{i}\right)}{2}\right|_{-1}^{2}+\frac{v}{2}\left\|\nabla w^{h, n+1 / 2}\right\|^{2} .
\end{aligned}
$$

Substituting the last bound we get

$$
\begin{aligned}
& \frac{1}{2 \Delta t}\left(\left|w^{h, n+1}\right|_{A}-\left|w^{h, n}\right|_{A}\right) \\
&+\frac{v}{2}\left\|\nabla w^{h, n+1 / 2}\right\|^{2}+v \delta^{2}\left\|\zeta^{h, n+1 / 2}\right\|^{2} \\
& \leq \frac{1}{2 v}\left|\frac{f\left(t_{n+1}\right)+f\left(t_{n}\right)}{2}\right|_{-1}^{2}
\end{aligned}
$$

Multiplying both sides by $2 \Delta t$ and summing over all time levels gives the desired result.

\section{Theorem 8 (Convergence)}

Let $w^{h, n+1}$ be computed by Algorithm 6. Let $f \in$ $L^{2}\left(0, T ; H^{-1}(\Omega)\right)$,

$$
\Delta t \leq v^{3}|| \nabla w||_{L^{4}\left(0, T ; L^{2}\right)^{\prime}}^{-4}
$$

$$
\begin{gathered}
w \in L^{2}\left(0, T ; H^{m+1}(\Omega)\right) \cap L^{4}\left(0, T ; H^{1}(\Omega)\right), \\
w_{t} \in L^{4}\left(0, T ; H^{1}(\Omega)\right),
\end{gathered}
$$

$w_{t t t} \in L^{2}\left(0, T ; H^{1}(\Omega)\right), q \in L^{2}\left(0, T ; H^{m}(\Omega)\right)$.

Then there exist a constant $C=C(\Omega, T, u, q, f, v)$, such that for $n=0, \ldots, M-1$,

$$
\begin{aligned}
& \| w^{M}-w^{h, M}||^{2}+\left.\delta^{2}|| \nabla\left(w^{M}-w^{h, M}\right)\right|^{2} \\
& +v \Delta t \sum_{n=0}^{M-1}|| \nabla\left(w^{n+1 / 2}-w^{h, n+1 / 2}\right) \|\left.\right|^{2} \\
& +v \delta^{2} \Delta t \sum_{n=0}^{M-1}|| \nabla\left(\zeta^{n+1 / 2}-\zeta^{h, n+1 / 2}\right)||^{2} \\
& \leq C v^{-1} \Delta t \sum_{n=0}^{M-1}\left[h^{2 m}+\delta^{4}+\Delta t^{4}\right] .
\end{aligned}
$$

Proof. We first set $v=v^{h}$ in (5), $\xi=\xi^{\text {h }}$ in (6) and $q=q^{h}$ in (7), and then take their averages of $n t h$ and $n+1$ st time levels.

$$
\begin{gathered}
\left(\frac{w_{t}^{n+1}+w_{t}^{n}}{2}, v^{h}\right)+\delta^{2}\left(\nabla\left(\frac{w_{t}^{n+1}+w_{t}^{n}}{2}\right), \nabla v^{h}\right) \\
+v\left(\nabla w^{n+1 / 2}, \nabla v^{h}\right)+v \delta^{2}\left(\nabla \zeta^{n+1 / 2}, \nabla v^{h}\right) \\
+b^{*}\left(w^{n+1 / 2}, w^{n+1 / 2}, v^{h}\right)-\left(q^{n+1 / 2}, \nabla \cdot v^{h}\right) \\
+\frac{\Delta t^{2}}{4} b^{*}\left(\frac{w^{n+1}-w^{n}}{\Delta t}, \frac{w^{n+1}-w^{n}}{\Delta t}, v^{h}\right) \\
=\left(\frac{f\left(t_{n+1}\right)+f\left(t_{n}\right)}{2}, v^{h}\right), \\
\left(\nabla w^{n+1 / 2}, \nabla \xi^{h}\right)=\left(\zeta^{n+1 / 2}, \xi^{h}\right)
\end{gathered}
$$




$$
\left(\nabla \cdot w^{n+1 / 2}, \chi^{h}\right)=0
$$

Then, subtracting (12) from (15) and (13) from (16) and letting $e^{\tau}=w^{\tau}-w^{h, \tau}$ give the following error equations:

$$
\begin{gathered}
\left(\frac{w_{t}{ }^{n+1}+w_{t}{ }^{n}}{2}-\frac{w^{h, n+1}-w^{h, n}}{\Delta t}, v^{h}\right) \\
+\delta^{2}\left(\nabla\left(\frac{w_{t}{ }^{n+1}+w_{t}{ }^{n}}{2}\right)-\nabla\left(\frac{w^{h, n+1}-w^{h, n}}{\Delta t}\right), \nabla v^{h}\right) \\
+v\left(\nabla e^{n+1 / 2}, \nabla v^{h}\right)+v \delta^{2}\left(\nabla\left(\zeta^{n+1 / 2}-\zeta^{h, n+1 / 2}\right), \nabla v^{h}\right) \\
+b^{*}\left(w^{n+1 / 2}, w^{n+1 / 2}, v^{h}\right)-b^{*}\left(w^{h, n+1 / 2}, w^{h, n+1 / 2}, v^{h}\right) \\
+\frac{\Delta t^{2}}{4} b^{*}\left(\frac{w^{n+1}-w^{n}}{\Delta t}, \frac{w^{n+1}-w^{n}}{\Delta t}, v^{h}\right) \\
-\left(q^{n+1 / 2}-q^{h, n+1 / 2}, \nabla \cdot v^{h}\right)=0, \\
\left(\nabla e^{n+1 / 2}, \nabla \xi^{h}\right)=\left(\zeta^{n+1 / 2}-\zeta^{h, n+1 / 2}, \xi^{h}\right) .
\end{gathered}
$$

By Taylor expansion, $\frac{w^{n+1}-w^{n}}{\Delta t}-\frac{w_{t}^{n+1}+w_{t}^{n}}{2}=\Delta t^{2} \rho^{n+1 / 2}$, where $\rho^{n+1 / 2}=\frac{w_{t t t}^{n+\frac{1}{2}}}{8}$. Therefore,

$$
\begin{aligned}
& \left(\frac{w_{t}^{n+1}+w_{t}^{n}}{2}-\frac{w^{h, n+1}-w^{h, n}}{\Delta t}, v^{h}\right) \\
& =\left(\frac{e^{n+1}-e^{n}}{\Delta t}, v^{h}\right)+\Delta t^{2}\left(\rho^{n+1 / 2}, v^{h}\right)
\end{aligned}
$$

and

$$
\begin{aligned}
& \left(\nabla\left(\frac{w_{t}^{n+1}+w_{t}^{n}}{2}\right)-\nabla\left(\frac{w^{h, n+1}-w^{h, n}}{\Delta t}\right), \nabla v^{h}\right) \\
& =\left(\nabla\left(\frac{e^{n+1}-e^{n}}{\Delta t}\right), \nabla v^{h}\right)+\Delta t^{2}\left(\nabla \rho^{n+1 / 2}, \nabla v^{h}\right)
\end{aligned}
$$

Decompose the error in two parts: $e^{\tau}=\eta^{\tau}-\psi^{h, \tau}$ where $\eta^{\tau}=w^{\tau}-\widetilde{w^{\tau}}$ and $\psi^{h, \tau}=w^{h, \tau}-\widetilde{w^{\tau}}$ and add and subtract $\widetilde{\zeta^{n+1 / 2}}$, where $\widetilde{w^{n+1} / 2} \in V^{h}, \widetilde{\zeta^{n+1 / 2}} \in X^{h}$ are chosen as the modified Stokes projection, defined via (9). Putting all these together and setting

$$
\begin{aligned}
& v^{h}=\psi^{h, n+1 / 2} \text { and } \xi^{h}=\zeta^{n+1 / 2}-\widetilde{\zeta^{n+1 / 2}} \text { yield } \\
& \left(\frac{\psi^{h, n+1}-\psi^{h, n}}{\Delta t}, \psi^{h, n+1 / 2}\right) \\
& \quad+\delta^{2}\left(\nabla\left(\frac{\psi^{h, n+1}-\psi^{h, n}}{\Delta t}\right), \nabla \psi^{h, n+1 / 2}\right)
\end{aligned}
$$

$$
\begin{gathered}
+v\left(\nabla \psi^{h, n+1 / 2}, \nabla \psi^{h, n+1 / 2}\right) \\
+v \delta^{2}\left(\nabla\left(\zeta^{h, n+1 / 2}-\zeta^{n+1 / 2}\right), \nabla \psi^{h, n+1 / 2}\right) \\
=\left(\frac{\eta^{n+1}-\eta^{n}}{\Delta t}, \psi^{h, n+1 / 2}\right) \\
+\delta^{2}\left(\nabla\left(\frac{\eta^{n+1}-\eta^{n}}{\Delta t}\right), \nabla \psi^{h, n+1 / 2}\right) \\
+\Delta t^{2}\left(\rho^{n+1 / 2}, \psi^{h, n+1 / 2}\right)+\Delta t^{2}\left(\nabla \rho^{n+1 / 2}, \nabla \psi^{h, n+1 / 2}\right) \\
+\frac{\Delta t^{2}}{4} b^{*}\left(\frac{w^{n+1}-w^{n}}{\Delta t}, \frac{w^{n+1}-w^{n}}{\Delta t}, \psi^{h, n+1 / 2}\right) \\
+b^{*}\left(w^{n+1 / 2}, w^{n+1 / 2}, \psi^{h, n+1 / 2}\right) \\
-b^{*}\left(w^{h, n+1 / 2}, w^{h, n+1 / 2}, \psi^{h, n+1 / 2}\right), \\
=\left(\left(\zeta^{h, n+1 / 2}-\widetilde{\zeta^{n+1 / 2}}\right),\left(\zeta^{h, n+1 / 2}-\widetilde{\zeta^{n+1 / 2}}\right)\right) .
\end{gathered}
$$

Since $\left(\widetilde{w^{n+1} / 2}, \widetilde{\zeta^{n+1 / 2}}\right)$ is taken to be the modified Stokes projection of $\left(w^{n+1 / 2}, \zeta^{n+1 / 2}\right) \in\left(\mathrm{V}^{\mathrm{h}}, \mathrm{X}^{\mathrm{h}}\right)$ some of the terms in the error equation disappears.

Substituting the second equation in the first and applying Cauchy-Schwartz inequality we get

$$
\begin{aligned}
& \frac{1}{2 \Delta t}\left(|| \psi^{h, n+1}||^{2}-|| \psi^{h, n}||^{2}\right) \\
& +\frac{\delta^{2}}{2 \Delta t}\left(|| \nabla \psi^{h, n+1}||^{2}-|| \nabla \psi^{h, n}||^{2}\right) \\
& +v|| \nabla \psi^{h, n+1 / 2}||^{2}+v \delta^{2}|| \zeta^{h, n+1 / 2}-\left.\widetilde{\zeta^{n+1 / 2}}\right|^{2} \\
& \leq|| \frac{\eta^{n+1}-\eta^{n}}{\Delta t}||_{-1}|| \nabla \psi^{h, n+1 / 2}|| \\
& +\delta^{2}|| \nabla \frac{\eta^{n+1}-\eta^{n}}{\Delta t}|||| \nabla \psi^{h, n+1 / 2}|| \\
& \Delta t^{2}|| \rho^{n+1 / 2}||_{-1}|| \nabla \psi^{h, n+1 / 2}|| \\
& +\Delta t^{2}|| \nabla \rho^{n+1 / 2}|||| \nabla \psi^{h, n+1 / 2}|| \\
& +\frac{\Delta t^{2}}{4} b^{*}\left(\frac{w^{n+1}-w^{n}}{\Delta t}, \frac{w^{n+1}-w^{n}}{\Delta t}, \psi^{h, n+1 / 2}\right) \\
& +b^{*}\left(w^{n+1 / 2}, w^{n+1 / 2}, \psi^{h, n+1 / 2}\right) \\
& -b^{*}\left(w^{h, n+1 / 2}, w^{h, n+1 / 2}, \psi^{h, n+1 / 2}\right) .
\end{aligned}
$$

The last two nonlinear terms on the right-hand side are decomposed as follows:

$\mid b^{*}\left(w^{h, n+1 / 2}, w^{h, n+1 / 2}, \psi^{h, n+1 / 2}\right)$ 


$$
\begin{gathered}
-b^{*}\left(w^{n+1 / 2}, w^{n+1 / 2}, \psi^{h, n+1 / 2}\right) \mid \\
=\left|b^{*}\left(\eta^{n+1 / 2}, w^{n+1 / 2}, \psi^{h, n+1 / 2}\right)\right| \\
+\left|b^{*}\left(\psi^{h, n+1 / 2}, w^{n+1 / 2}, \psi^{h, n+1 / 2}\right)\right| \\
+\left|b^{*}\left(w^{h, n+1 / 2}, \eta^{n+1 / 2}, \psi^{h, n+1 / 2}\right)\right| .
\end{gathered}
$$

Applying the upper bounds on trilinear forms followed by Young's inequalities

$$
\begin{aligned}
& \left|b^{*}\left(\eta^{n+1 / 2}, w^{n+1 / 2}, \psi^{h, n+1 / 2}\right)\right| \\
& \leq C|| \nabla \eta^{n+1 / 2}|||| \nabla w^{n+1 / 2}|||| \nabla \psi^{h, n+1 / 2} \| \mid \\
& \leq\left.\mu|| \nabla \psi^{h, n+1 / 2}\right|^{2}+\frac{C}{\mu}|| \nabla \eta^{n+1 / 2}||^{2}|| \nabla w^{n+1 / 2}||^{2} \\
& \left|b^{*}\left(\psi^{h, n+1 / 2}, w^{n+1 / 2}, \psi^{h, n+1 / 2}\right)\right| \\
& \leq C|| \psi^{h, n+1 / 2}||^{1 / 2}|| \nabla \psi^{h, n+1 / 2}||^{3 / 2}|| \nabla w^{n+1 / 2}||^{2} \\
& \leq \mu|| \nabla \psi^{h, n+1 / 2}||^{2}+\frac{C}{\mu^{3}}|| \nabla w^{n+1 / 2}||^{4}|| \psi^{h, n+1 / 2}||^{2}, \\
& \left|b^{*}\left(w^{h, n+1 / 2}, \eta^{n+1 / 2}, \psi^{h, n+1 / 2}\right)\right| \\
& \leq C|| \nabla \eta^{n+1 / 2}|||| \nabla w^{h, n+1 / 2}|||| \nabla \psi^{h, n+1 / 2}||^{2} \\
& \leq\left.\mu|| \nabla \psi^{h, n+1 / 2}\right|^{2}+\frac{C}{\mu}|| \nabla \eta^{n+1 / 2}||^{2}|| \nabla w^{h, n+1 / 2}||^{2}
\end{aligned}
$$

Also, for the first nonlinear term,

$$
\begin{aligned}
& \frac{\Delta t^{2}}{4} b^{*}\left(\frac{w^{n+1}-w^{n}}{\Delta t}, \frac{w^{n+1}-w^{n}}{\Delta t}, \psi^{h, n+1 / 2}\right) \\
& \leq \frac{\mathrm{C} \Delta t^{2}}{4}\left\|\nabla \frac{w^{n+1}-w^{n}}{\Delta t}||^{2}|| \nabla \psi^{h, n+1 / 2}\right\| \mid \\
& \leq\left.\mu|| \nabla \psi^{h, n+1 / 2}\right|^{2}+\mathrm{C} \Delta t^{4}|| \nabla \frac{w^{n+1}-w^{n}}{\Delta t}||^{4}
\end{aligned}
$$

On the right-hand side of (3.7), we apply Young's inequality and choose an appropriate $\mu$ to get

$$
\begin{aligned}
& \frac{1}{2 \Delta t}\left(|| \psi^{h, n+1}||^{2}-|| \psi^{h, n}||^{2}\right) \\
& +\frac{\delta^{2}}{2 \Delta t}\left(|| \nabla \psi^{h, n+1}||^{2}-|| \nabla \psi^{h, n}||^{2}\right) \\
& +\left.v|| \nabla \psi^{h, n+1 / 2}\right|^{2}+v \delta^{2}|| \zeta^{h, n+1 / 2}-\widehat{\zeta^{n+1 / 2}}||^{2} \\
& \leq\left. C v^{-1}|| \frac{\eta^{n+1}-\eta^{n}}{\Delta t}\right|_{-1} ^{2}+C v^{-1} \delta^{4}|| \nabla \frac{\eta^{n+1}-\eta^{n}}{\Delta t}||^{2}
\end{aligned}
$$

$$
\begin{array}{r}
+C v^{-1} \Delta t^{4}|| \rho^{n+1 / 2}||_{-1}^{2}+C v^{-1} \Delta t^{4}|| \nabla \rho^{n+1 / 2}||^{2} \\
+C v^{-1}|| \nabla \eta^{n+1 / 2}||^{2}\left(\left.|| \nabla w^{n+1 / 2}\right|^{2}+|| \nabla w^{h, n+1 / 2}||^{2}\right) \\
+C \Delta t^{4}|| \nabla \frac{w^{n+1}-w^{n}}{\Delta t}||^{4} \\
+\left.C v^{-3}|| \nabla w^{n+1 / 2}||^{4}|| \psi^{h, n+1 / 2}\right|^{2} .
\end{array}
$$

Multiplying both sides by $2 \Delta t$ and summing over all time levels,

$$
\begin{aligned}
& || \psi^{h, M}||^{2}+\delta^{2}|| \nabla \psi^{h, M}||^{2} \\
& +\left.v \Delta t \sum_{n=0}^{M-1}|| \nabla \psi^{h, n+1 / 2}\right|^{2} \\
& +v \delta^{2} \Delta t \sum_{n=0}^{M-1}|| \zeta^{h, n+1 / 2}-\left.\widetilde{\zeta^{n+1 / 2}}\right|^{2} \\
& \leq|| \psi^{h, 0}\left\|^{2}+\delta^{2}|| \nabla \psi^{h, 0}\right\|^{2} \\
& +C v^{-1} \Delta t \sum_{n=0}^{M-1}\left[|| \frac{\eta^{n+1}-\eta^{n}}{\Delta t}||_{-1}^{2}+\delta^{4}|| \nabla \frac{\eta^{n+1}-\eta^{n}}{\Delta t}||^{2}+\right. \\
& \Delta t^{4}\left(|| \rho^{n+1 / 2}||_{-1}^{2}+|| \nabla \rho^{n+1 / 2}||^{2}+|| \nabla \frac{w^{n+1}-w^{n}}{\Delta t}||^{4}\right)+ \\
& \left.|| \nabla \eta^{n+1 / 2}||^{2}\left(|| \nabla w^{n+1 / 2}||^{2}+|| \nabla w^{h, n+1 / 2}||^{2}\right)\right] \\
& +\Delta t \sum_{n=0}^{M-1}\left[C v^{-3}|| \nabla w^{n+1 / 2}||^{4}|| \psi^{h, n+1 / 2}||^{2}\right] \text {. }
\end{aligned}
$$

Use the approximation properties of $X^{h}, Q^{h}$. Since the mesh nodes do not depend upon the time level, it follows from (10) and (11) that

$$
\begin{aligned}
& \Delta t \sum_{i=0}^{n} \|\left.\frac{\eta^{n+1}-\eta^{n}}{\Delta t}\right|_{-1} ^{2} \leq\left. C \Delta t \sum_{i=0}^{n}|| \frac{\eta^{n+1}-\eta^{n}}{\Delta t}\right|^{2} \\
& \leq C h^{2 m} \\
& \Delta t \sum_{i=0}^{n}\left\|\nabla \frac{\eta^{n+1}-\eta^{n}}{\Delta t}\right\|_{-1}^{2} \leq\left. C \Delta t \sum_{i=0}^{n}\left\|\nabla \frac{\eta^{n+1}-\eta^{n}}{\Delta t}\right\|\right|^{2} \\
& \leq C h^{2 m} \\
& k \sum_{i=0}^{n}|| \nabla \eta^{n+1 / 2} \|^{2} \leq C h^{2 m} .
\end{aligned}
$$

Under the assumption of the theorem 


$$
\begin{aligned}
& \Delta t \sum_{i=0}^{M-1} \Delta t^{4}|| \rho^{n+1 / 2}\left\|_{-1}^{2} \leq C \Delta t \sum_{i=0}^{n} \Delta t^{4}|| \rho^{n+1 / 2}\right\|^{2} \\
& \leq C \Delta t^{4}, \\
& \Delta t \sum_{i=0}^{n} \Delta t^{4}|| \nabla \rho^{n+1 / 2} \|^{2} \leq C \Delta t^{4}, \\
& \Delta t \sum_{i=0}^{M-1} \Delta t^{4}\left\|\nabla \frac{w^{n+1}-w^{n}}{\Delta t}\right\|^{4} \leq C \Delta t^{4} .
\end{aligned}
$$

Applying the discrete Gronwall's lemma and using bounds in the stability and the accuracy of the modified stokes projection theorem, give

$$
\begin{aligned}
|| \psi^{h, M} \|^{2}+ & \delta^{2}|| \nabla \psi^{h, M}\left\|^{2}+v \Delta t \sum_{n=0}^{M-1}|| \nabla \psi^{h, n+1 / 2}\right\|^{2} \\
& +v \delta^{2} \Delta t \sum_{n=0}^{M-1} \| \zeta^{h, n+1 / 2}-\left.\widetilde{\zeta^{n+1 / 2}}\right|^{2} \\
\leq & C v^{-1}\left[h^{2 m}+\delta^{4}+\Delta t^{4}\right] .
\end{aligned}
$$

It must be noted that initial $\left\|\psi^{h, 0}\right\|^{2}+\delta^{2}|| \nabla \psi^{h, 0} \|^{2}$ er-

rors stays within the desired accuracy due to the preposi-

tion 4 of the modified Stokes projection.

The stated error estimate now follows by applying the triangle inequality.

\section{Corollary 9 (Optimal Convergence Rate)}

Suppose that in addition to the assumptions of the above theorem, $X^{h}$ and $Q^{h}$ are composed of Taylor-Hood finite elements (P2 - P1). Then the error is of the form, ||$w^{M}-w^{h, M}||$

$$
\begin{gathered}
+\left[v \Delta t \sum_{n=0}^{M-1}|| \nabla\left(w^{n+1 / 2}-w^{h, n+1 / 2}\right) \|^{2}\right]^{1 / 2} \\
\leq \mathcal{O}\left(h^{2}+\delta^{2}+\Delta t^{2}\right) .
\end{gathered}
$$

\section{Numerical Results}

First, we perform a convergence test for the proposed $\mathrm{CN}-\mathrm{ADM}$. In this test non-homogeneous Dirichlet boundary conditions are implemented. Although theory of the model requires periodicity, this choice of boundary conditions still performs as Corollary (9) suggests. Computations are performed with deal.II - a generalpurpose object-oriented finite element library [17] - on a machine running with Ubuntu 16.04.4 LTS operating system.
For the convergence rate assessment, consider a twodimensional problem with a known exact solution of the NSE in

$$
\begin{gathered}
\quad \Omega=[0,1]^{2} \text { by } \\
u_{1}=e^{-t}\left(1-x^{2}-y^{2}\right) y, \\
u_{2}=e^{-t}\left(x^{2}+y^{2}-1\right) x, \\
p=0 .
\end{gathered}
$$

The forcing $f$ and initial condition $u_{0}$ are computed so that it complies with (1). The final time in the computations is $T=1$. In order to verify the theoretical claims on the convergence rates, we take

$$
\Delta t=h=2 \delta=2^{-N} \text {. }
$$

For $v=0.1$ the convergence rates in Table 1 confirm what is predicted by Corollary (9): the convergence rates of $2^{\text {nd }}$ order are achieved. Also, we observe the asymptotic character of convergence, which is typical for the ADM methods, $[8,9,11,12]$.

To save horizontal space in the following tables, let the error norm $\left\|u-w^{h}\right\|_{L^{2}\left(0, T ; L^{2}(\Omega)\right)}$ and $\left\|u-w^{h}\right\|_{L^{2}\left(0, T ; H^{-1}(\Omega)\right)}$ be abbreviated to $\| u-$ $u^{h} \|_{L^{2} L^{2}}$ and ||$u-u^{h} \|_{L^{2} H^{1}}$, respectively.

Table 1. Errors and convergence rates for $\mathbf{v}=\mathbf{0 . 1}$

\begin{tabular}{c|c|c|c|c}
$\mathrm{N}$ & ||$u-u^{h}||_{L^{2} L^{2}}$ & $\mathrm{CR}$ & ||$u-\left.u^{h}\right|_{L^{2} H^{1}}$ & $\mathrm{CR}$ \\
\hline 2 & $1.29135 \mathrm{e}-03$ & - & $2.33857 \mathrm{e}-02$ & - \\
\hline 3 & $6.04768 \mathrm{e}-04$ & 1.09 & $6.70917 \mathrm{e}-03$ & 1.80 \\
\hline 4 & $2.08182 \mathrm{e}-04$ & 1.54 & $1.97635 \mathrm{e}-03$ & 1.76 \\
\hline 5 & $5.61673 \mathrm{e}-05$ & 1.89 & $5.23328 \mathrm{e}-04$ & 1.92 \\
\hline 6 & $1.42303 \mathrm{e}-05$ & 1.98 & $1.32449 \mathrm{e}-04$ & 1.98
\end{tabular}

As the viscosity coefficient $v=10^{-5}$ decreases, the convergence rates improve slower for $\left\|u-w^{h}\right\|_{L^{2}\left(0, T ; H^{-1}(\Omega)\right)}$

norm - see the results for the flow at $v=10^{-5}$ in Table 2. Similar phenomenon is also observed in [12].

For the qualitative assessment, consider the 2D flow past an obstacle Figure 1, see [14]- [16]. In this setting the channel height is $H=0.41 \mathrm{~m}$, the channel length is $L=2.2 \mathrm{~m}$, the diameter of the circle whose center is located at $0.2 \mathrm{~m}$ above the lower wall and $0.2 \mathrm{~m}$ right of the left wall of the channel is $D=0.1 \mathrm{~m}$. 


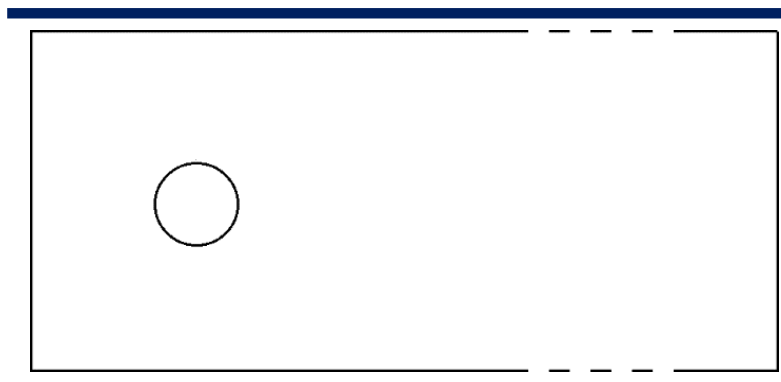

Fig. 1. Flow Domain turbulence model; targeted to solve a variation of NSE not the NSE itself.

Table 3. Comparison of drag, lift coefficients and pressure difference estimations with various references.

\begin{tabular}{c|c|c|c|c} 
Ref. & {$[16]$} & {$[16]$} & {$[15]$} & CN-ADM \\
\hline$\Delta t$ & 0.001 & 0.001 & 0.00125 & 0.01 \\
\hline dof & 14,446 & 56,477 & 399,616 & 16,626 \\
\hline$C_{D, \max }$ & 2.2844 & 2.8472 & 2.95092 & 2.70686 \\
\hline$C_{L, \max }$ & 0.0176 & 0.4010 & 0.47795 & 0.44425 \\
\hline$\Delta P$ & -0.1267 & -0.1138 & -0.1116 & -0.1152
\end{tabular}

Table 2. Errors and convergence rates for $\mathbf{v}=\mathbf{0 . 0 0 0 0 1}$

\begin{tabular}{c|c|c|c|c}
$\mathrm{N}$ & ||$u-u^{h}||_{L^{2} L^{2}}$ & $\mathrm{CR}$ & ||$u-\left.u^{h}\right|_{L^{2} H^{1}}$ & $\mathrm{CR}$ \\
\hline 2 & $7.59320 \mathrm{e}-03$ & - & $6.28074 \mathrm{e}-02$ & - \\
\hline 3 & $3.21435 \mathrm{e}-03$ & 1.24 & $3.05891 \mathrm{e}-02$ & 1.04 \\
\hline 4 & $9.57501 \mathrm{e}-04$ & 1.75 & $1.39632 \mathrm{e}-02$ & 1.13 \\
\hline 5 & $2.50309 \mathrm{e}-04$ & 1.94 & $6.72520 \mathrm{e}-03$ & 1.05 \\
\hline 6 & $6.26949 \mathrm{e}-05$ & 2.00 & $2.87011 \mathrm{e}-03$ & 1.23
\end{tabular}

Initially flow velocity and pressure are chosen zero everywhere in the domain. 'No slip' boundary conditions on the horizontal walls are strongly enforced. The time dependent inflow/outflow boundary condition is set to

$$
w(0, y, t)=w(2.2, y, t)=6 y(0.41-y) /(0.41)^{2} .
$$

Choosing $v=0.001$, this construction results in a time dependent Reynolds number, $0 \leq \operatorname{Re}(t) \leq 100$.

The correct behavior for this setting is, from time $t=$ $2 \mathrm{~s}$ to $\mathrm{t}=4 \mathrm{~s}$, two vortices start to develop behind the obstacle, and then they separate, later on a vortex street forms which can be visible until the final time $t=8$, see [15],[16]. Plots of the flow at $t=2,4,6$ and 8 are shown in Figure 2. These results look comparable with their corresponding plots in [15]-[16].

We also compute the maximum drag $\left(\mathrm{C}_{\mathrm{D}}\right)$ and lift $\left(\mathrm{C}_{\mathrm{L}}\right)$ coefficients along with the pressure differences $\Delta P$ as in [15] with Taylor-Hood elements. The filter radius $\delta$ is chosen to be the average mesh width. It must be noted that the proposed CN-ADM algorithm is a
Also, our computations are performed on a very coarse mesh with a higher time step-size. Therefore, an exact mateh with the reference values of lift and drag is not required. Nevertheless, we should observe results close by these values to consider CN-ADM useful. In this regard, we mostly compare our results with those obtained in [16].

Table 3 suggests that $\mathrm{CN}-\mathrm{ADM}$ performs quite better than the Leray model (without any indicator given in [16]) solved with 14,446 degrees of freedom. Comparing to the finer-mesh results with Leray model, CN-ADM seems to produce better results for lift coefficient even if it was computed 10 times coarse time step size and around 3.4 times less degrees of freedom. For the drag coefficient and pressure drop, Leray model with higher degrees of freedom produces better results.

\section{Conclusions}

A turbulence model with high accuracy both in temporal and spatial variables presented in this report. Both theory and computational results suggest that proposed high accuracy is indeed achieved. Also, a qualitative testing is presented that match with its reference turbulent behavior. In addition to these, computations for drag and lift coefficients of a benchmark problem is presented: illustrating this model could be employed for such computations beside any qualitative assessments. 

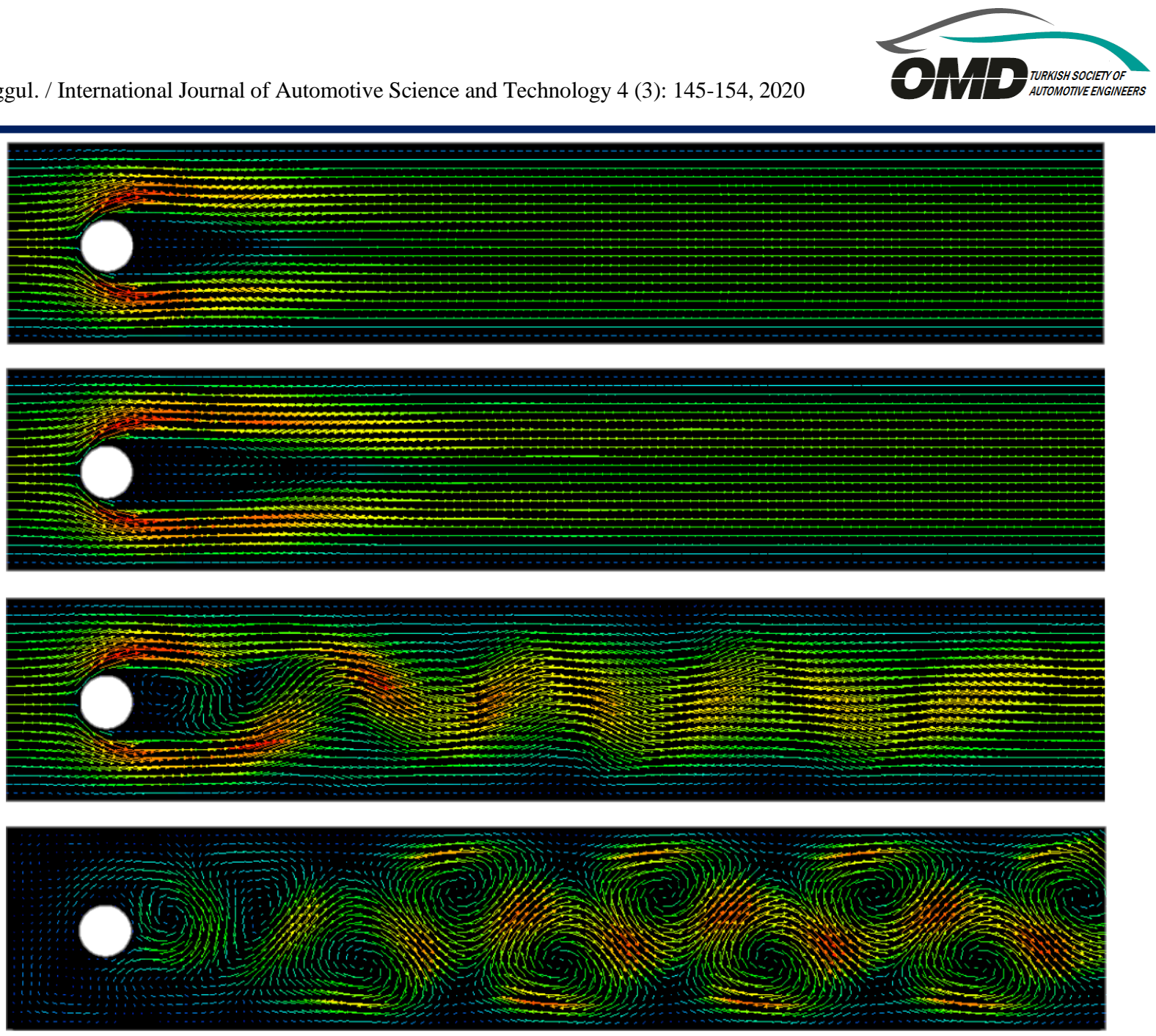

Fig. 2. Flow Profiles at Time $\mathbf{t}=\mathbf{2 , 4}, \mathbf{6}, \mathbf{8 s}$.

\section{References}

[1] Sagaut, P. (2006). Large eddy simulation for incompressible flows. Scientific Computation, Springer Verlag.

[2] John, V. (2004). Large eddy simulation of turbulent incompressible flows. Lecture Notes in Computational Science and Engineering, Springer Verlag.

[3] Berselli, L.C. and Iliescu, T., and Layton, W.J. (2006). Mathematics of large eddy simulation of turbulent flows. Scientific Computation, Springer Verlag.

[4] Adams, N.A. and Stolz, S. (2001). Deconvolution methods for subgrid-scale approximation in large-eddy simulation. Modern Simulation Strategies for Turbulent Flow, B. Geurts (editor), pp. 21-41, R. T. Edwards.

[5] Adams, N.A., Stolz, S., Kleiser, L. (2006). The Approximate deconvolution model for compressible flows: isotropic turbulence and shock-boundary-layer interaction. Fluid Mechanics and Its Applications. Advances in LES of Complex Flows, vol. 65, 2006, pp. 33-47, Springer Netherlands.

[6] Cardoso Manica, C., and Merdan, S. K. (2007). Finite ele- ment error analysis of a zeroth order approximate deconvolution model based on a mixed formulation. JMAA, vol. 331, no. 1, pp. 669-685.

[7] Layton, W. and Lewandowski, R. (2006). Residual stress of approximate deconvolution models of turbulence. Journal of Turbulence, vol. 7, Issue 46, p. 1-21.

[8] Labovsky, A., Trenchea, C. (2011). Large eddy simulation for turbulent magnetohydrodynamic flows. JMMA, vol. 377, pp.516-533.

[9] Labovsky, A., Trenchea, C. (2010). A family of approximate deconvolution models for magneto hydrodynamic turbulence. Numerical Functional Analysis and Optimization, vol.31(12), pp.1362-1385.

[10]Dunca, A. and Epshteyn, Y. (2006). On the Stolz-Adams deconvolution model for the large eddy simulation of turbulent flows. SIAM J. Math. Anal., Vol. 37(6), pp. 1890-1902.

[11]Gunzburger, M., Labovsky A. (2012). High accuracy method for turbulent flow problems. Mathematical Models and Methods in Applied Sciences, vol. 22 (6).

[12]Aggul, M., Kaya, S., Labovsky, A. (2019). Two approaches to creating a turbulence model with increased temporal accu- 
racy. Applied Mathematics and Computations, vol. 358, pp. 25-36.

[13]Girault, V., Raviart, P.A. (1979). Finite element approximation of the Navier-Stokes equations. Lecture notes in mathematics, no. 749, Springer-Verlag.

[14]Schäfer M., Turek S., Durst F., Krause E., Rannacher R. (1996). Benchmark computations of laminar flow around a cylinder. Flow Simulation with High-Performance Computers II. Notes on Numerical Fluid Mechanics (NNFM), vol 48. Vieweg+Teubner Verlag.

[15]John, V. (2004). Reference values for drag and lift of a two dimensional time-dependent flow around a cylinder. International Journal for Numerical Methods in Fluids, 44:777-788.

[16]Bowers, A. L., Rebholz, L.G., Takhirov A., Trenchea C. (2012). Improved accuracy in regularization models of incompressible flow via adaptive nonlinear filtering. Int. J. Numer. Meth. Fluids, 70: 805-828.

[17]Bangerth, W., Hartmann R., Kanschat G. (2007). deal.II - A general purpose object oriented finite element library. ACM Trans. Math. Software 33 (4) 24/1-24/27. 